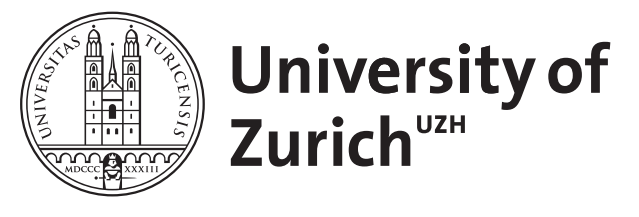

\title{
Interleukin-2: biology, design and application
}

\author{
Arenas-Ramirez, Natalia ; Woytschak, Janine ; Boyman, Onur
}

\begin{abstract}
Interleukin-2 (IL-2) exerts crucial functions during immune homeostasis via its effects on regulatory T (Treg) cells, and the optimizing and fine-tuning of effector lymphocyte responses. Thus, somewhat paradoxically, low doses of recombinant IL-2 have been used for Treg cell-based immunosuppressive strategies against immune pathologies, while high-dose IL-2 has shown some success in stimulating antitumor immune responses. Recent studies of the functional, biophysical and structural characteristics of IL-2 have led to the generation of IL-2 formulations, including IL- $2 / \mathrm{mAb}$ complexes and IL-2 variants (muteins) that selectively enhance IL-2's immune stimulatory versus inhibitory properties. Here, we review these findings, placing new mechanistic insights into improved next-generation IL-2 formulations within the broader context of IL-2 biology. We conclude by integrating these findings into a framework for understanding IL-2-mediated selective immune modulation.
\end{abstract}

DOI: https://doi.org/10.1016/j.it.2015.10.003

Posted at the Zurich Open Repository and Archive, University of Zurich

ZORA URL: https://doi.org/10.5167/uzh-123112

Journal Article

Published Version

Originally published at:

Arenas-Ramirez, Natalia; Woytschak, Janine; Boyman, Onur (2015). Interleukin-2: biology, design and application. Trends in Immunology, 36(12):763-777.

DOI: https://doi.org/10.1016/j.it.2015.10.003 


\section{Interleukin-2: Biology, Design and Application}

\author{
Natalia Arenas-Ramirez, ${ }^{1,2}$ Janine Woytschak, ${ }^{1,2}$ and \\ Onur Boyman ${ }^{1, *}$
}

Interleukin-2 (IL-2) exerts crucial functions during immune homeostasis via its effects on regulatory $\mathrm{T}$ (Treg) cells, and the optimizing and fine-tuning of effector lymphocyte responses. Thus, somewhat paradoxically, low doses of recombinant IL-2 have been used for Treg cell-based immunosuppressive strategies against immune pathologies, while high-dose IL-2 has shown some success in stimulating anti-tumor immune responses. Recent studies of the functional, biophysical and structural characteristics of IL-2 have led to the generation of IL-2 formulations, including IL-2/mAb complexes and IL-2 variants (muteins) that selectively enhance IL-2's immune stimulatory versus inhibitory properties. Here, we review these findings, placing new mechanistic insights into improved next-generation IL-2 formulations within the broader context of IL-2 biology. We conclude by integrating these findings into a framework for understanding IL-2-mediated selective immune modulation.

\section{Introduction}

Discovered, isolated, and cloned between 1976 and 1983, IL-2 was the first immunotherapy demonstrating clinical efficacy in metastatic cancer [1-5]. Despite these promising data, IL-2 immunotherapy has not been widely adopted for various reasons, including its difficult administration due to its short in vivo half-life $\left(T_{1 / 2}\right)$, its toxic adverse effects when administered at high doses (as needed for antitumor immunotherapy), and its ability to stimulate both cytotoxic effector $T$ cells and regulatory $T$ (Treg) cells [6]. While activation of Treg cells is an unwanted effect in anticancer IL-2 immunotherapy, as Treg cells can dampen effector $T$ cell responses against tumor antigens, the property of IL-2 - even at low doses - to stimulate Treg cells could be harnessed for the treatment of Treg cell-deficient autoimmune and chronic inflammatory disorders [7].

The past 10 years rekindled an interest in IL-2 immunotherapy, which came from structural and biophysical insights into the complex formed by IL-2 with its IL-2 receptor (IL-2R) subunits [8] and the finding that IL-2 could be modified to selectively stimulate either cytotoxic effector $T$ cells or Treg cells [9]; these studies have led to the generation of IL-2 formulations with improved and selective immune stimulatory capacities [10]. Furthermore, clinical trials using low-dose IL-2 have demonstrated IL-2's potential in expanding Treg cells and modulating immune pathologies $[11,12]$. Four recent publications exemplify this development and have motivated this review, in that they provide structural insight into the selective IL-2-mediated modulation of immune responses using IL-2/monoclonal antibody (mAb) complexes [13], highlight the possibility of using IL-2 muteins to antagonize, rather than stimulate, IL-2R-induced signals [14], and report novel data on the clinical use of low-dose IL-2 therapy in organ-specific and systemic autoimmune disease $[15,16]$.

To place these findings in context, we begin by providing an introduction into the biology of IL-2 and its receptors, followed by a discussion of IL-2/mAb complexes and IL-2 muteins, and end by

\section{Trends}

Interleukin-2 (IL-2) exerts immunosuppressive and immunostimulatory effects by activating regulatory $T$ (Treg) versus cytotoxic effector cells

These IL-2 effects hinge on different IL-2 receptor (IL-2R) expression patterns: $\mathrm{CD}^{+} \mathrm{T}$ and natural killer cells carry high levels of dimeric IL-2Rs comprising IL-2R $\beta$ (CD122) and IL-2R $\gamma\left(\gamma_{c}\right)$; Treg cells express high IL-2R $\propto$ (CD25) levels along with intermediate levels of CD122 and $\gamma_{\mathrm{c}}$.

Selective IL-2 formulations, such as IL-2 complexes and IL-2 muteins, preferentially stimulate cells expressing high CD25 versus high CD122 levels, and recent studies extend these concepts to also include muteins that inhibit IL-2R-induced responses.

These data converge into a framework of IL-2-mediated selective immune modulation where CD25-biased IL-2 formulations primarily expand Treg cells and CD122-directed IL-2 formulations stimulate cytotoxic effector cells.

'Department of Immunology, University Hospital Zurich, University of Zurich, $\mathrm{CH}-8091$ Zurich, Switzerland

${ }^{2}$ These authors contributed equally.

onur.boyman@uzh.ch (O. Boyman). 
summarizing the key concepts arising from these studies as well as indicating gaps in our current understanding.

\section{The Biology of IL-2}

IL-2 is a 15.5-16-kDa, four- $\propto$-helix-bundle cytokine (Figure 1) that exerts its actions via binding to various IL-2Rs, notably monomeric, dimeric, and trimeric IL-2Rs $[6,17,18]$. Monomeric IL2Rs, comprising IL-2R (CD25), are usually cell membrane associated but also exist in soluble form and bind IL-2 with a low $K_{d}$ of $\sim 10^{-8} \mathrm{M}$. Interaction of IL-2 with CD25 alone does not induce a signal [19]; hence, isolated membrane-bound or soluble CD25 molecules might serve as scavenger or decoy receptors for IL-2 $[6,20]$. Conversely, both dimeric and trimeric IL-2Rs lead to a downstream signal on binding to IL-2. Dimeric IL-2Rs comprise IL-2R $\beta$ (CD122) and IL2R $\gamma$ [better known as common $\gamma$-chain $\left(\gamma_{c}\right)$ or CD132], whereas trimeric IL-2Rs comprise CD25, CD122, and $\gamma_{c}$ (Figure 2). Of note, CD122 is also part of IL-15R, whereas $\gamma_{c}$ is shared by IL-2, IL4, IL-7, IL-9, IL-15, and IL-21 [18]. Considering only IL-2Rs with signaling capacity, dimeric IL2Rs can be referred to as low-affinity $\left(K_{d} \sim 10^{-9} \mathrm{M}\right)$ and trimeric IL-2Rs as high-affinity ( $K_{d}$ $\sim 10^{-11} \mathrm{M}$ ) IL-2Rs [19]. On a molecular level, a single trimeric IL-2R binds IL-2 with roughly 10100 -fold higher affinity than a single dimeric IL-2R, illustrating that one function of CD25 is to improve IL-2 binding to the dimeric IL-2R. Association of IL-2 with IL-2R causes internalization of the quaternary complex, on which IL-2, CD122, and $\gamma_{\mathrm{c}}$ become degraded in vesicles, whereas CD25 is recycled via endosomes to the cell surface [21]. Notably, internalization of IL-2 has been suggested to depend on the cytoplasmic part of $\gamma_{c}$, suggesting that $\gamma_{c}$-mediated signaling is involved [22].

On triggering of IL-2R, signal transduction occurs via three major pathways, involving: (i) Janus kinase (JAK)-signal transducer and activator of transcription (STAT); (ii) phosphoinositide 3kinase (PI3K)-AKT; and (iii) mitogen-activated protein kinase (MAPK) [6,18] (see Figure 2 for more details). Moreover, IL-2 signaling activates the transcription factor B lymphocyte-induced maturation protein 1 (Blimp1), encoded by Prdm1, which serves as a negative feedback loop by repressing IL-2 production.

Several immune cells have been shown to secrete IL-2 when activated, including $T$ cell receptor (TCR) $\propto \beta^{+}$and TCR $\gamma \delta^{+}$T cells, natural killer (NK) cells, NKT cells, dendritic cells (DCs), and mast cells [6]. At resting conditions, $\mathrm{CD}^{+}$helper $\mathrm{T}$ (Th) cells are the main source of the constant but low levels of IL-2. On immune activation, IL-2 production rapidly rises. Low IL-2 secretion by activated DCs has been suggested to provide an early IL-2 source [23], thereby supporting T cell stimulation. In parallel, activated T cells, including $\mathrm{CD} 4^{+}$and $\mathrm{CD}^{+} \mathrm{T}$ cells, start secreting large amounts of IL-2 for their own (autocrine) use and to stimulate in a paracrine fashion neighboring IL-2R ${ }^{+}$cells $[6,17,18]$. Interestingly, Treg cells are unable to produce IL-2, even on stimulation, unless they constitute peripherally derived ('induced') Treg cells redifferentiating to Th cells [24]. IL-2 production by activated $\mathrm{T}$ cells is transient and transcriptionally regulated, including silencing by Blimp1 and Aiolos (encoded by Ikzf3) [18]. Moreover, negative feedback appears to also exist on the cellular level involving IL-2-producing CD4 ${ }^{+} \mathrm{T}$ and IL-2-consuming Treg cells [25]. Such regulation mechanisms might be key in preventing $T$ cell overstimulation by persistent IL-2 signals in conjunction with repetitive TCR stimulation by antigen (including self- and tumor antigens), which can lead to T cell exhaustion or Fas (CD95)-mediated activation-induced cell death $[26,27]$.

In terms of IL-2 responsiveness, in vitro activated human T cells have been reported to carry about 2000 high-affinity and 11000 low-affinity IL-2Rs per cell [4,28]. Dimeric IL-2Rs are expressed at high levels on antigen-experienced (memory) $C D 8^{+} \mathrm{T}$ cells and NK cells, whereas low to intermediate levels of dimeric IL-2Rs are found on memory CD4 ${ }^{+}$and naive T cells [6]. On TCR stimulation, T cells transiently upregulate CD25, thus expressing now trimeric IL-2Rs. In 
(A) MYRMQLISCIALSLALVTNS
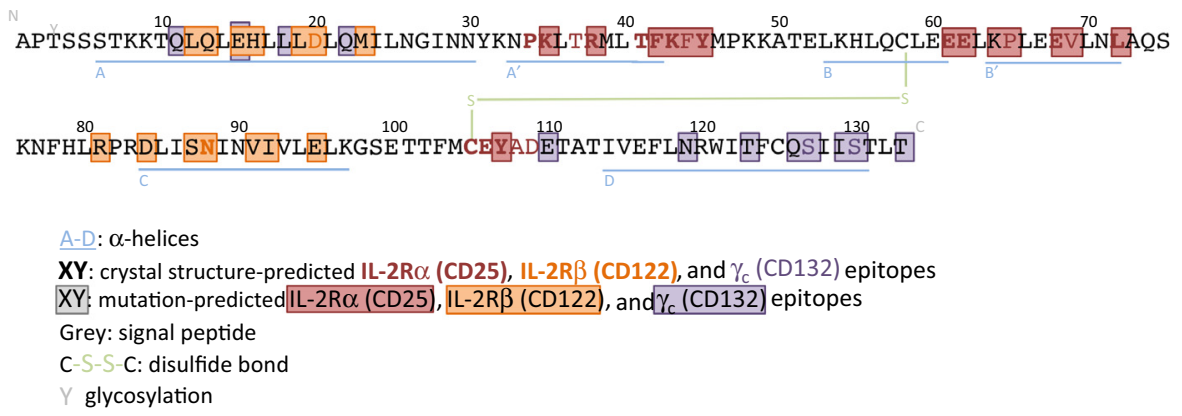

(B)

CD25 epitopes

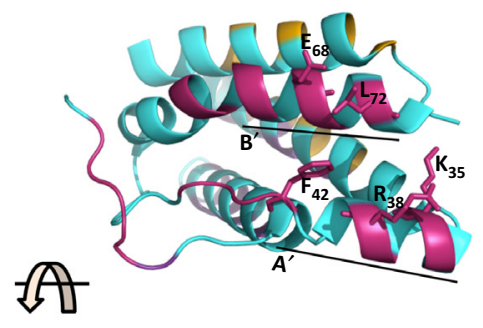

CD122 epitopes
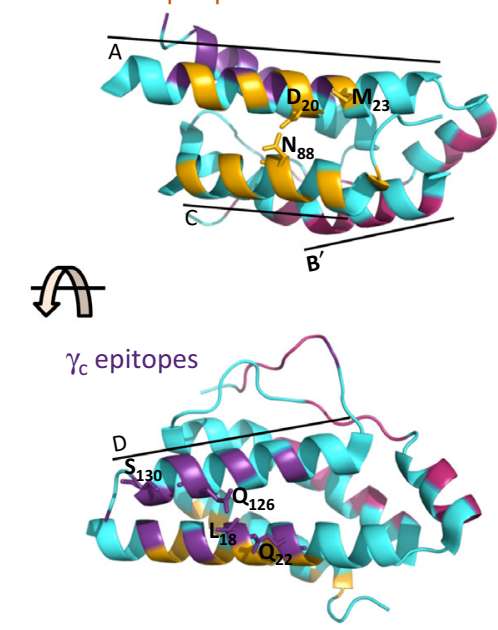

(C)

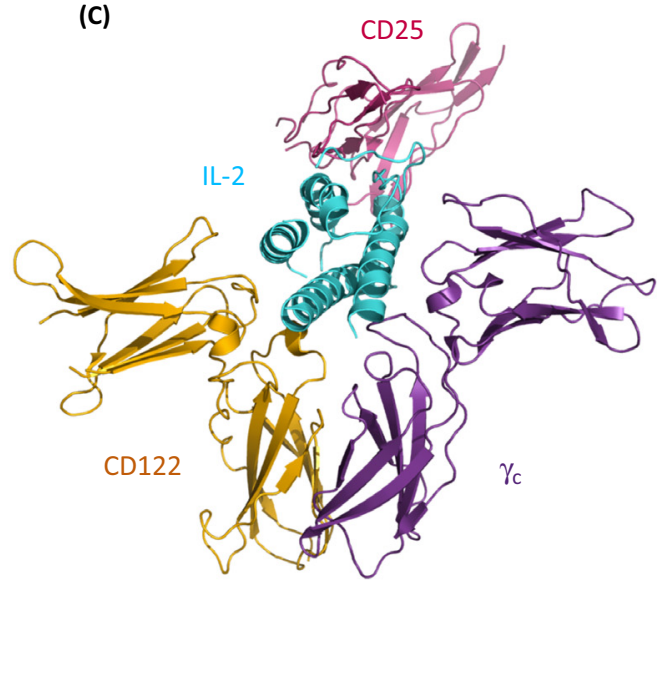

Trends in Immunology

Figure 1. Sequence and Structure of IL-2 in Association with its Receptor Subunits. (A) Amino acid sequence of IL-2, indicating amino acids that interact with IL-2 receptor (IL-2R) subunits, including IL-2R (CD25; pink), IL-2R (CD122; orange), and common $\gamma$-chain ( $\gamma_{c}$, CD132; purple). Human IL-2 comprises 133 amino acids and weighs $15.5 \mathrm{kDa}$; mouse IL-2 measures 149 amino acids and has 16 kDa molecular mass. Human and mouse IL-2 show $57 \%$ sequence homology, yet human IL-2 can efficiently stimulate mouse IL-2Rs whereas mouse IL-2 is rather inefficient in binding to human IL-2Rs. (B) Human IL-2 (light blue) and its epitopes for CD25 (pink), CD122 (orange), and $\gamma_{\mathrm{c}}$ (purple) (accession code PDB: 1M47 [82]). (C) 3D structure of the quaternary IL-2-IL-2R complex, comprising IL-2 (light blue), CD25 (pink), CD122 (orange), and $\gamma_{c}$ (purple) (accession code PDB: 2B5I [8]).

addition to recently activated T cells, high levels of trimeric IL-2Rs (precisely, high CD25 levels plus intermediate levels of dimeric IL-2Rs) are found constitutively on thymus-derived ('natural') $\mathrm{CD}^{+}$forkhead box p3 (Foxp3) ${ }^{+}$Treg cells [29], whereas type 2 innate lymphoid cells (ILC2) and certain nonimmune cells (such as pulmonary endothelial cells) have been reported to carry low to 
(A) $\mathrm{APC}$

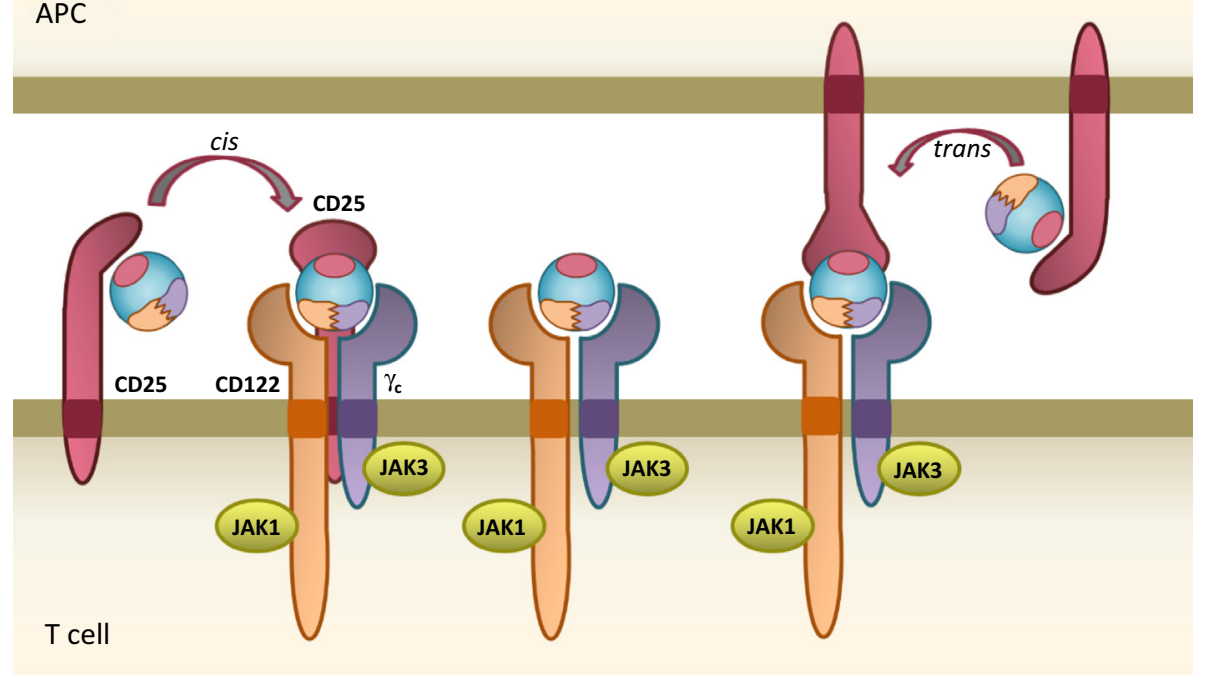

(B)

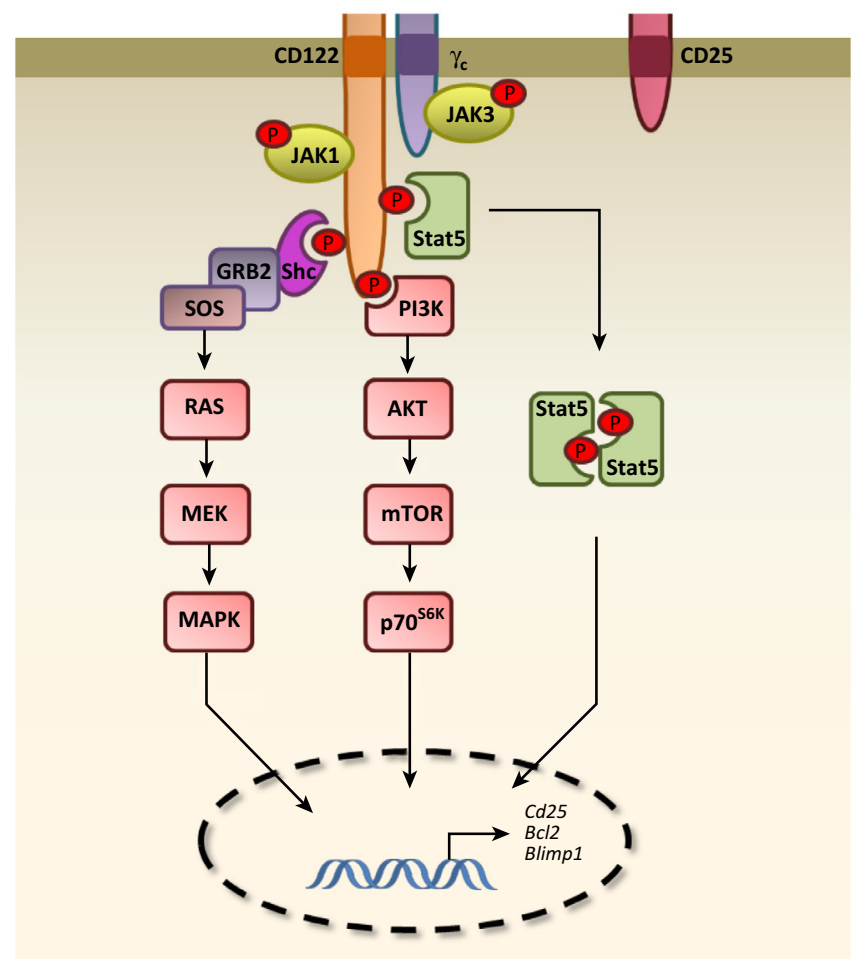

Trends in Immunology

Figure 2. IL-2 Receptor (IL-2R) Binding and Signaling. Cartoon of IL-2 interacting with its receptor subunits, including $\mathrm{IL}-2 \mathrm{R} \propto(\mathrm{CD} 25), \mathrm{IL}-2 \mathrm{R} \beta$ (CD122), and the common $\gamma$-chain $\left(\gamma_{\mathrm{c}}, \mathrm{CD} 132\right)$, as well as signaling pathways following the interaction of IL-2 with various IL-2R subunits. Binding of IL-2 to CD122 and $\gamma_{C}$ causes heterodimerization of the cytoplasmic tails of these receptor subunits and activation of Janus kinase 1 (JAK1) and JAK3 (associated with CD122 and $\gamma_{c}$ respectively) [18]. Activated JAK1 and JAK3 exert kinase activity on key tyrosine (Y) residues of CD122, which subsequently allows recruitment of the adaptor protein SHC and of STAT1, STAT3, and STAT5 (including STAT5A and STAT5B), Phosphorylated STAT5A and STAT5B then oligomerize forming STAT5 dimers and tetramers before undergoing nuclear translocation, where they bind to key target genes responsible for cell activation, differentiation, and proliferation. SHC in turn serves as a platform for activating the Ras-Raf-MEK-ERK mitogen-activated protein kinase (MAPK) pathway. Additionally, IL-2R triggering activates the phosphoinositide 3-kinase (PI3K)-AKT-mammalian target of rapamycin (mTOR)-p70 S6 kinase pathway. 
very low levels of trimeric IL-2Rs [30,31]. Furthermore, activated mouse and human B cells express functional trimeric IL-2Rs and proliferate in vitro on stimulation with human IL-2 (hIL-2) $[32,33]$. Interestingly, DCs have been reported to express CD25 and to use CD25 to present IL-2 in trans to T cells expressing dimeric IL-2Rs [23,34] (Figure 2). Whether DCs carry CD122 is controversial, and stronger evidence suggests that DCs do not express CD122 under steadystate conditions [6,34].

The low but constant IL-2 production during resting conditions serves primarily for the development and homeostatic survival of Treg cells, which in turn maintain peripheral immune tolerance by dampening (autoreactive) effector $T$ cells $[15,17,35]$. Treg cell dependence on IL-2 signaling is maybe best illustrated in IL-2- or IL-2R subunit (CD25 or CD122)-deficient animals and patients, which all lack Foxp3 ${ }^{+}$Treg cells and develop systemic autoimmunity and inflammatory bowel disease. In IL-2R-deficient mice, this pathology can be prevented by adoptive transfer of wild-type Treg cells [36,37]. Following immune stimulation, local IL-2 concentrations increase several-fold, especially in secondary lymphoid organs, causing proliferation of NK cells as well as memory and recently activated effector T cells. Timed IL-2 signals are also required for optimal primary and secondary $C D 8^{+} T$ cell responses $[6,38,39]$ as well as for effector-to-memory transition in $\mathrm{CD}^{+} \mathrm{T}$ cells [40]. Furthermore, IL-2 in concert with polarizing cytokines (pcs) (in parentheses) facilitates differentiation of naive Th cells to interferon- $\gamma$-producing Th1 (pc: IL-12), IL-4-, IL-5-, IL-9-, and IL-13-secreting Th2 (pc: IL-4), and peripherally derived ('induced') Treg [pc: transforming growth factor- $\beta$ (TGF- $\beta$ )] cells, while conversion of Th cells to IL-17A-, IL-17F-, and IL-22-producing Th17 (pcs: IL-6 and TGF- $\beta$ ) and follicular Th (pcs: IL-6 and IL-21) cells is curtailed by IL-2 signaling $[6,18]$.

According to the current view, low doses of IL-2 [i.e., 1500 000-3 000000 international units (IU) once daily in humans or 15000-30 $000 \mathrm{IU}$ or lower once daily in mice] preferentially stimulate Treg cells, although some effector T and NK cells might become activated as well [7]. Low-dose IL-2 might thus be suitable for the treatment of autoimmune and chronic inflammatory diseases such as systemic lupus erythematosus (SLE), type 1 diabetes, and cryoglobulinemic vasculitis [11,15,16,41,42], as well as graft rejection and chronic graft-versus-host disease [12,43], as these conditions have been reported to often feature lower IL-2 signaling and a relative Treg to effector T cell deficiency [7]. In line with this, a recent study showed, in patients with SLE, a relative reduction of Treg to effector $\mathrm{T}$ cells, with Treg cells displaying lower CD25 levels; moreover, cultured (but unstimulated) peripheral blood mononuclear cells and $\mathrm{CD} 4^{+} \mathrm{T}$ cells from SLE patients showed deficient ex vivo IL-2 production [16]. Administration of a 5-day course of low-dose IL-2 to five SLE patients led to preferential stimulation of Treg over effector T cells, thus restoring Treg to effector T cell balance [16], as also observed in other pathologies $[7,43]$. According to a recent publication on human Treg cells from healthy controls and patients with type 1 diabetes, Treg cells seem to be especially poised to sense low concentrations of IL-2, as assessed by measuring phosphorylated STAT5, in part due to their high expression of CD25 and $\gamma_{c}$ as well as their further transcriptional upregulation of CD25 and Foxp3 rapidly on IL-2; conversely, about tenfold higher IL-2 concentrations were needed to induce STAT5 phosphorylation in other lymphocytes, with memory $\mathrm{CD}^{+} \mathrm{T}$ cells and $\mathrm{CD} 56^{\text {high }} \mathrm{NK}$ cells being the nextmost-sensitive subsets, followed by naive $\mathrm{CD} 4^{+}$and memory $\mathrm{CD} 8^{+} T$ cells and naive $\mathrm{CD} 8^{+} \mathrm{T}$ and CD56 ${ }^{\text {low }}$ NK cells [15].

Conversely, high-dose IL-2 (i.e., 600 000-720 $000 \mathrm{IU} / \mathrm{kg}$ body weight three times daily for up to 14 doses per cycle in humans or $100000 \mathrm{IU}$ or higher once or twice daily in mice) has been used for immunotherapy against metastatic cancer [5] as high doses of IL-2 stimulate antitumor cytotoxic lymphocytes, including effector T and NK cells, presumably, once trimeric IL-2Rs on Treg cells have been saturated with IL-2. High-dose IL-2 treatment leads to 15-19\% objective clinical response, including 7-9\% complete response, in patients with metastatic melanoma or 
metastatic renal cell carcinoma [5]. Other diseases where IL-2 immunotherapy has been tried comprise inherited and acquired immune deficiencies, the latter including chronic HIV infection, and other viral infections [44-46]. As an alternative to low-dose versus high-dose IL-2, various selective IL-2 formulations have been generated, including IL-2/mAb complexes and mutant IL2 molecules (IL-2 muteins), which are discussed in the next sections.

\section{IL-2/mAb Complexes}

IL-2/mAb complexes (also termed IL-2 complexes or IL-2/anti-IL-2 mAb complexes) consist of IL-2 plus a particular anti-IL-2 mAb. The anti-IL-2 mAb can bind to and potentiate endogenous or recombinant IL-2 $[6,9]$.

Two functionally distinct IL-2/mAb complexes are distinguished [6,9,47]. Use of the anti-mouse IL-2 (mlL-2) mAb S4B6 (i.e., mAb clone S4B6-1 or other S4B6-like mAbs, including JES6-5H4 for mIL-2 and MAB602 for hIL-2) generates IL-2/mAb complexes that preferentially stimulate cells expressing high levels of dimeric IL-2Rs, most notably memory (and memory-phenotype) $\mathrm{CD}^{+} \mathrm{T}$ cells and NK cells. Conversely, IL-2/mAb complexes comprising IL-2 plus the anti-mIL-2 mAb JES6-1 (i.e., mAb clone JES6-1A12 or anti-hlL-2 mAb 5344) selectively activate cells carrying high levels of CD25 in addition to CD122 and $\gamma_{\mathrm{c}}$. For example, five to seven daily injections of IL-2/S4B6 complexes (using 15 $000 \mathrm{IU}$ IL-2) expand memory CD8 ${ }^{+} \mathrm{T}$ cells and NK cells by 20-40-fold, while on such treatment $\mathrm{CD} 25^{+}$Foxp $3^{+}$Treg cell counts increase by two- to fivefold only $[9,31,47]$. This selectivity correlates with CD122 expression on memory CD8 ${ }^{+} \mathrm{T}$ and NK cells (both CD122 ${ }^{\text {high}}$ ) versus Treg cells (expressing intermediate levels of CD122). Moreover, by avoiding contact with $\mathrm{CD} 25^{+}$endothelial cells and by reducing the IL-2 dose needed for clinical efficacy, IL-2/S4B6 complexes exert less endothelial cell damage and vascular leak syndrome (VLS) in the lungs and liver of treated animals [31] (see also the section on IL-2 muteins). Given these properties, IL-2/S4B6 complexes could be used therapeutically in metastatic malignancies and chronic viral infection. In support of this notion, several groups have reported favorable results using IL-2/S4B6 complexes either as a monotherapy or in combination with another agent (such as Toll-like receptor ligand, agonist anti-OX40 mAb, or peptide vaccine) in various cancer models, including B16 melanoma, Lewis lung carcinoma, MC38 colon carcinoma, MCA-205 sarcoma, and TRAMP-C1 prostate carcinoma [31,48-53]. Moreover, IL-2/S4B6 complexes have shown efficacy in models of acute and chronic infection $[54,55]$.

For IL-2/JES6-1 complexes, a short course of three to seven daily injections of these complexes increases $\mathrm{CD}_{25} 5^{+}$Foxp3 $^{+}$Treg cell numbers by $7-15$-fold, while $\mathrm{CD} 8^{+} \mathrm{T}$ and NK cells are not significantly affected by IL-2/JES6-1 complexes $[9,47,56]$. IL-2/JES6-1 complexes are also stimulatory for ILC2 that express trimeric IL-2Rs, which contributes to IL-5 production and eosinophilia $[30,57]$. IL-2/JES6-1 complexes have shown promising results in the prevention of pancreatic [56] and skin [58] allograft rejection as well as in the treatment of several autoimmune and inflammatory diseases in mice, including type 1 diabetes in nonobese diabetic mice [59], experimental autoimmune encephalomyelitis (a model of multiple sclerosis) [56], experimental myasthenia [60], collagen-induced arthritis [61], dextran sodium sulfate-induced acute colitis [13], and T cell-mediated allergic airway disease [62]. Interestingly, administration of IL-2/JES6-1 complexes also improved the pathology of some metabolic, cardiovascular, and degenerative disorders, such as murine obesity-induced inflammation and insulin resistance ('type 2 diabetes') [63], atherosclerosis in high-fat diet-fed apolipoprotein E-deficient animals [64], and the mdx mouse model of Duchenne muscular dystrophy [65], that feature inflammatory infiltrates and are thus amenable to suppression by $\mathrm{CD} 25^{+}$Foxp $3^{+}$Treg cells.

Mechanistically, IL-2/mAb complexes are characterized, compared with IL-2, by increased in vivo potency and selectivity toward CD122 ${ }^{\text {high }}$ versus $\mathrm{CD} 25^{\text {high }}$ cells. Regarding their enhanced 
(A)

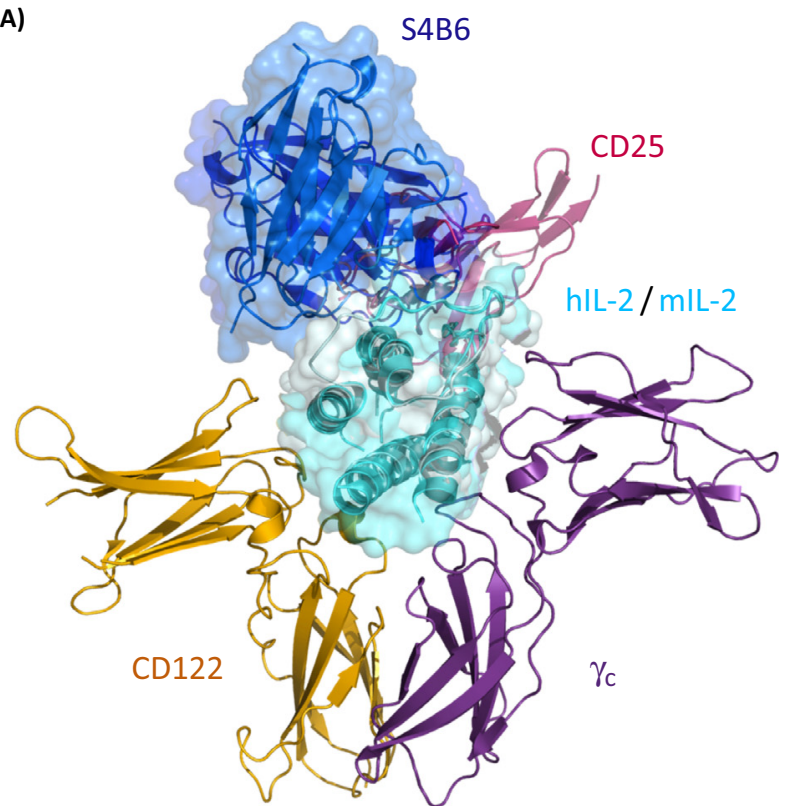

(B)

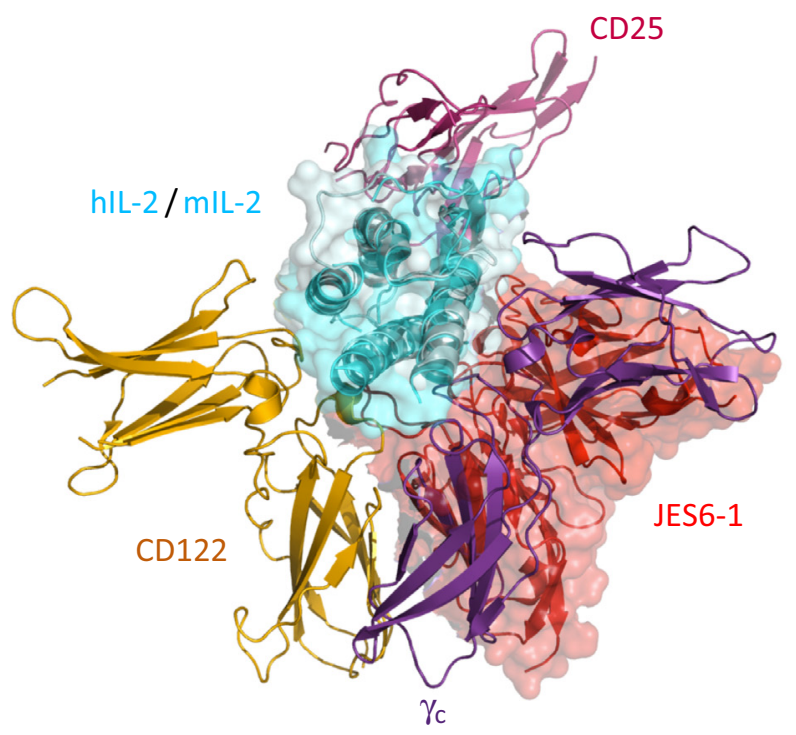

Figure 3. 3D Structure of IL-2/mAb Complexes. (A,B) The complexes formed by mouse IL-2 with Fab fragments of anti-mouse IL-2 monoclonal antibody (A) S4B6 (clone S4B6-1; accession code PDB: 4YUE [13]) or (B) JES6-1 (clone JES6-1A12; accession code PDB: 4YQX [13]) overlaying the human IL-2IL-2R complex (accession code PDB: 2B5I [8]).

\footnotetext{
Trends in Immunology

Treng

potency, IL-2/mAb complexes display an increased biological $T_{1 / 2}$ compared with IL-2 [47]. Unmodified IL-2 is rather short lived on intravenous injection ( $T_{1 / 2} \sim 3-5 \mathrm{~min}$ in mice and $7-10 \mathrm{~min}$ in humans) due to its rapid clearance in the kidneys, probably by degradation, followed by delayed clearance [66-68]. The association of IL-2 with larger molecules, including serum albumin, liposomes, and irrelevant mAbs or mAb fragments (i.e., unrelated mAbs specific for antigens other than IL-2), increases the lifespan of IL-2 [69]. Similarly, association of IL-2 with S4B6 or JES6- 1 increases IL-2 $T_{1 / 2}$ by about 10-20-fold $[47,70]$. This $T_{1 / 2}$ increase depends on neonatal FC receptors and is crucial for the activity of IL-2/JES6- 1 complexes and, to a lesser extent, IL-2/S4B6 complexes; contrarily, Fc receptors for IgG (FCR $\gamma)$ do not appear to play a significant role in the bioactivity of IL-2/mAb complexes $[47,70]$. Nevertheless, similar to
} 
CD25-mediated trans-presentation of IL-2 by DCs to T cells [34], IL-2/mAb complexes might bind to FcRys on DCs and become presented in trans to T cells, although this has not been formally proven. Of note, the anti-IL-2 mAbs belong to various IgG subclasses - with S4B6 being rat IgG2a, JES6-5H4 rat IgG2b, JES6-1 rat IgG1, MAB602 mouse IgG2a, and 5344 mouse lgG1 $[9,47]$ - suggesting that, for these mAbs, subclass plays a subordinate role.

Another mechanism for the superagonistic in vivo activity of IL-2/mAb complexes could be enhanced IL-2R signaling due to crosslinking of two dimeric IL-2Rs by an IL-2 complex, structural changes in IL-2 on mAb binding, or prolonged interaction of IL-2 with its receptor. IL-2R crosslinking has so far not been formally disproved. Yet, compared with IL-2, testing of IL2/S4B6 complexes in vitro on CD25 ${ }^{-}$YT-1 human NK cells induced decreased STAT5 phosphorylation [13] and lower proliferation of wild-type and CD25 ${ }^{-1-}$ memory-phenotype mouse $\mathrm{CD}^{+} \mathrm{T}$ cells [9]. These data would rather argue against IL-2R crosslinking by IL-2/ S4B6 complexes. For IL-2/JES6-1 complexes, STAT5 phosphorylation was reduced in CD25 ${ }^{+}$ YT-1 human NK cells compared with IL-2 in vitro [13], although another report using in vitro stimulation of total mouse splenocytes suggested that, compared with IL-2, IL-2/JES6-1 complexes led to increased STAT5 phosphorylation in CD4 ${ }^{+}$T cells [71]. In line with the former finding, activation of $\mathrm{CD}_{2} 5^{+}$murine $\mathrm{CD} 8^{+} \mathrm{T}$ cells in vitro led to about tenfold lower proliferation with IL-2/JES6-1 complexes compared with IL-2 [9]. Conversely, in vivo IL-2/JES6-1 complexes led to prolonged and increased phosphorylation of STAT5 in Treg cells compared with IL-2 [61].

In its simplest embodiment, a mechanistic model for the selectivity of different IL-2/mAb complexes should explain the following observations [6,9]: (i) IL-2/mAb complexes are formed using in vitro 'neutralizing' anti-IL-2 mAbs; (ii) S4B6 and JES6-1 bind to different IL-2 sites; (iii) IL2/S4B6 complexes mediate CD122-dependent but CD25-independent responsiveness of cells; and (iv) stimulation of cells by IL-2/JES6-1 complexes is strictly CD25 dependent. Based on functional in vivo and in vitro data, it has been proposed that the different functional properties of IL-2/S4B6 and IL-2/JES6-1 complexes are a result of the mAbs binding to different IL-2Rbinding sites of IL-2 [6,9]: association of IL-2 with S4B6 sterically impedes binding to CD25 by covering IL-2's CD25 epitope, thus biasing IL-2/S4B6 complexes to preferentially stimulate CD122 high cells, including memory $\mathrm{CD}^{+} \mathrm{T}$ and NK cells; conversely, complexing IL-2 with JES6-1 occludes IL-2's CD122 and/or $\gamma_{c}$ epitope, thus rendering IL-2 dependent on contact with CD25 and directing IL-2/JES6-1 complexes to selectively stimulate CD25 $5^{\text {high }}$ cells, such as Treg cells.

Recent structural data not only provide evidence for the steric aspects of the abovementioned model but also suggest mild antagonistic and allosteric forces at work (Figure 3). Thus, the data show that S4B6 covers CD25-interacting epitopes on IL-2, particularly those that lie on helices B and $\mathrm{C}$ of IL-2, which harbor some of the residues important for CD25 binding (Figure 1). However, S4B6 does not appear to contact the mouse counterparts of hlL-2 residues K35 and R38 (cf. mlL-2/S4B6 complex structure accessed at accession code PDB: 4YUE [13]). Both K35 and R38 have previously been shown to be intimately involved in the association of hlL-2 with human CD25 (Figure 1), with R38 representing the residue with the largest estimated buried surface with CD25 and most numerous van der Waals and hydrogen-bond contacts with CD25 [72]. Hence, these data suggest that S4B6's footprint on IL-2 does not fully overlap with that of CD25.

Moreover, S4B6 affected the binding of IL-2 to its receptor in two additional ways. Firstly, based on structural comparison of the mlL-2/S4B6 complex with unbound hlL-2 and hlL-2 in complex with human CD25, S4B6 binding caused $\mathrm{mLL}-2$ to undergo a slight conformational change in its helix C leading to increased affinity to CD122 [13], similar to what has been reported for human CD25-bound IL-2 [8]. Evidence for the relevance of this conformational change comes from the 
study of H9 and D10 hlL-2 muteins (also termed IL-2 'superkines'; see next section), which structurally replicated the said change in helix $C$ and showed 200-fold increased affinities to CD122, led to more STAT5 phosphorylation in CD25- YT-1 human NK cells, and enhanced proliferation of human naive $\mathrm{CD} 4^{+} \mathrm{T}$ cells in vitro [51]. In line with this hypothesis, immobilized monovalent mIL-2/S4B6 complex showed six-times-higher affinity to monomeric mouse CD122 compared with immobilized mIL-2 [13], which, however, is considerably lower than the binding affinity to CD122 of the IL-2 superkines [51]. Furthermore, the authors reported a small steric clash between S4B6 and CD122, presumably antagonizing, at least in part, the affinity-enhancing effects described above [13]. Nevertheless, the net result of these somewhat opposing effects was increased binding of IL-2/S4B6 to CD122; however, surprisingly, this did not translate into enhanced STAT5 phosphorylation [13] or T cell proliferation in vitro [9], which is unlike the case of IL-2 superkines.

Based on the recent crystal structure of the IL-2/JES6-1 complex, steric effects at the interfaces of IL-2 with CD122/ $\gamma_{C}$ and with CD25, as well as allosteric effects affecting IL-2's CD25-binding site, have been put forward [13]. The data demonstrated that hydrogen bond contacts existed between mIL-2 residues Q36 and E37 (corresponding to hIL-2 residues Q22 and M23; Figure 1) and JES6-1 [13], thereby causing steric competition between JES6-1 and CD122 and $\gamma_{c}$, respectively. While these aspects of the structure confirm the previously postulated model, the proposed steric competition between JES6-1 and CD25 and allosteric changes in IL-2's CD25 epitope are both unexpected and somewhat counterintuitive, considering that IL-2/JES6-1 complexes preferentially stimulate CD25+ cells. Thus, the authors reported that JES6- 1 distorted the entire CD25-binding $\mathrm{AB}$ loop of mlL-2 by contacting $\mathrm{mlL}-2$ residues $\mathrm{K} 49$ and R52, which in hlL-2 correspond to residues K35 and R38, which are central to CD25 interaction. This latter finding makes it difficult to understand how IL-2/JES6-1 complexes are still able to bind to and stimulate $\mathrm{CD}_{25} 5^{+}$cells, especially considering the low-affinity interaction of IL-2 with CD25 compared with the estimated 10-100-fold higher affinity of JES6-1 to IL-2. However, the authors offered a model whereby, in cells with very high CD25 expression levels, CD25 displaces JES6-1 from IL-2 by mass action, thus liberating IL-2 to engage CD122/ $\gamma_{\mathrm{C}}$. This effect is further enhanced by a positive feedback loop involving IL-2 signaling-mediated transcriptional upregulation of CD25 on responding cells $[6,13,73]$ (cf. also Figure 2).

Collectively, the recent structural data confirm the steric aspects of the proposed model and further suggest allosteric effects that, especially in IL-2/S4B6 complexes, appear to fine-tune the mechanistic steps of action of IL-2/mAb complexes, which allows the formulation of a framework for achieving selective IL-2 immunotherapy (discussed in more detail in the Concluding Remarks). In addition to IL-2/mAb complexes, studies on selective IL-2 muteins lend support for such a model, as outlined below.

\section{IL-2 Muteins}

An alternative approach to selective IL-2-mediated stimulation is the generation of IL-2 muteins. Initially, research in this area was driven by the search for IL-2 muteins with reduced toxic adverse effects. At that time, IL-2-induced toxic effects were thought to rely primarily on activated NK cells secreting proinflammatory cytokines, such as tumor necrosis factor- $\alpha$, and vasoactive mediators, which in turn led to endothelial damage and VLS [69]. To this end, IL-2 muteins were generated with decreased binding affinity to CD122, such as BAY 50-4798 (containing an N88R mutation of IL-2; see also Figure 1) and Selectikine (harboring a D20T mutation of IL-2) [74,75]. However, when tested in clinical trials such IL-2 muteins did not show decreased toxicity in patients $[10,76]$. More recent data showed that NK cells are implicated in VLS; however, the more proximal events of endothelial cell damage and VLS include the direct binding of IL-2 to CD25 $5^{+}$pulmonary endothelial cells [31]. Thus, use of IL-2/ S4B6 complexes or of the IL-2 muteins R38E, R38G, R38W, and F42A (all predicted or shown 
to impair binding to CD25) disfavors contact with endothelial cells and significantly reduces VLS $[10,31,77]$.

Capitalizing on these findings, more recent efforts aimed at generating IL-2 muteins with decreased affinity to CD25 (such as 'no- $\propto$ mutein' [78], GA501, and GA504 [79]). An alternative approach was taken based on the structural data of hIL-2 bound to CD25 [72] and the human quaternary $\mathrm{IL}-2-\mathrm{IL}-2 \mathrm{R}$ complex [8], which suggested that binding to CD25 led to the

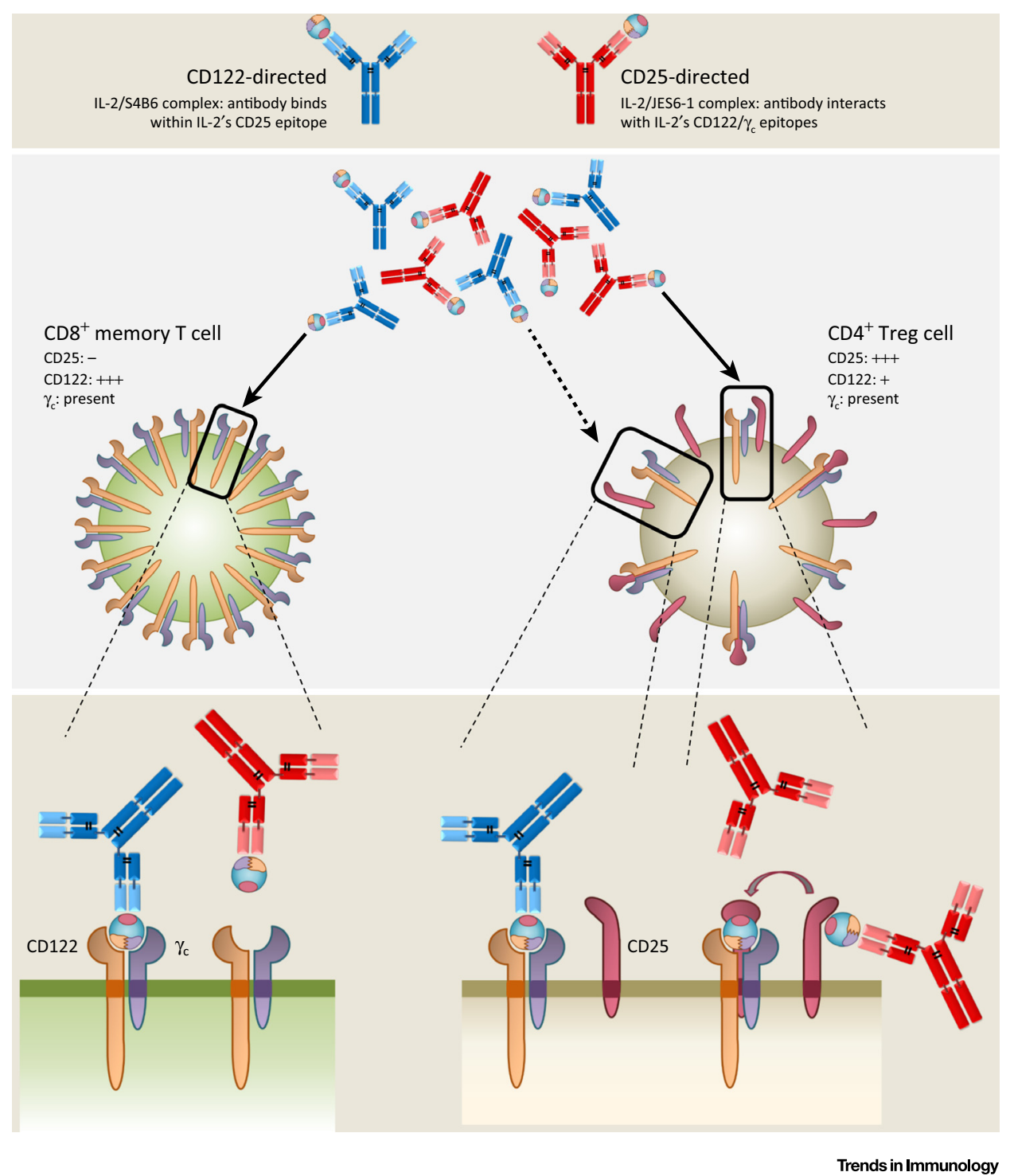

Figure 4. Framework for IL-2-Based Selective Immune Modulation, as Exemplified by IL-2/mAb Complexes. Selective immune modulation - that is, immune stimulation versus immune suppression - can be achieved using IL-2 that has been modified to (temporarily) impede IL-2's binding to certain IL-2R subunits. For example, in IL-2/S4B6 complexes (blue) the anti-IL-2 antibody binds to IL-2's CD25-binding epitope, thereby rendering IL-2 'blind' to CD25. For this and other reasons (see text), IL-2/S4B6 complexes preferentially stimulate cells expressing high levels of CD122 (in addition to $\gamma_{c}$, which in this model is assumed to be not limiting), including memory $\mathrm{CD}^{+} \mathrm{T}$ cells (green) and natural killer cells (not shown). $\mathrm{CD4}^{+}$regulatory $\mathrm{T}$ (Treg) cells (brown) express only intermediate levels of CD122, which is one of the reasons (see text) why IL-2/S4B6 complexes are less efficient in activating Treg cells. Conversely, in IL-2/JES6-1 complexes (red), the anti-IL-2 antibody interacts with IL-2's CD122 and $\gamma_{c}$ epitopes. As a consequence IL-2/JES6-1 complexes crucially depend on the presence of high levels of CD25, such as in CD25+ Treg cells. 
aforementioned structural change of helix C of IL-2 that improved its binding to CD122. Based on this hypothesis, the agonistic IL-2 muteins H9 and D10 (also known as IL-2 superkines) were generated [51]. These IL-2 muteins associated with CD122 with 200-fold increased affinity and efficiently bound dimeric IL-2Rs without the need for CD25; such binding resulted in increased STAT5 phosphorylation and cell proliferation in vitro and in vivo [51]. Use of the H9 IL-2 mutein showed increased $\mathrm{CD} 8^{+} \mathrm{T}$ cell-mediated antitumor activity against solid murine tumors [51] and enhanced NK cell-mediated cytotoxicity against MHC class I-deficient RMA-S tumor cells in vivo [80].

Leveraging on the properties of $\mathrm{H} 9$, an $\mathrm{H} 9$ mutant that retained CD122 binding but demonstrated significantly decreased affinity to $\gamma_{c}$ was engineered and characterized recently [14]. This was achieved by introducing four mutations into H9 (L18R, Q22E, Q126T, and S130R) and the resultant mutein was called H9-RETR. These newly introduced mutations of H9-RETR affect IL2 residues involved in binding to $\gamma_{c}$ (Figure 1). Interestingly, L18 and Q22 are interspaced by two residues, L19 and D20, that participate in CD122 binding, as shown by structural data [8] or the D20T IL-2 mutein Selectikine displaying reduced CD122 binding [75]; however, an H9-RE mutein containing only L18R and Q22E mutations did not show decreased signaling activity compared with H9 [14]. When used in vivo, an injection of H9-RETR-Fc4 (i.e., H9-RETR fused to human IgG4 FC domain) was able to block IL-2- or IL-15-induced STAT5 phosphorylation of murine $\mathrm{CD}^{+}{ }^{+} \mathrm{CD}_{25} 5^{+}$Foxp3 $^{+}$Treg cells. Moreover, a 10-day course of H9-RETR-Fc4 prevented acute graft-versus-host disease in a full MHC-mismatch C57BL/6 to BALB/c situation, presumably by blocking the IL-2R-mediated stimulation of activated effector T cells. In vitro, H9RETR was of comparable efficacy to daclizumab, an antihuman CD25 mAb, in inhibiting the proliferation of adult $\mathrm{T}$ cell leukemia cells.

Hence, engineered IL-2 muteins provide interesting tools in being able to either enhance or dampen IL-2-mediated responses and might be useful for IL-2-directed immune modulation.

\section{Concluding Remarks}

Taken together, the findings on IL-2/mAb complexes and IL-2 muteins suggest a mechanistic framework for IL-2-based selective immunotherapy, as exemplified by the two functionally different IL-2/mAb complexes (Figure 4). This concept hinges on the use of qualitatively different IL-2 variants, namely CD122-biased versus CD25-directed IL-2 formulations, rather than different IL-2 doses, such as high- versus low-dose IL-2 immunotherapy. IL-2/mAb complexes have served as a paradigm for IL-2-based selective immune modulation. But how accurate is our mechanistic understanding of $\mathrm{LL}-2 / \mathrm{mAb}$ complexes?

A mechanistic model of IL-2/mAb complexes, including the new elements arising from the recent biophysical and structural data, needs to consider the following aspects [6,9,13]: (i) IL-2/mAb complexes comprise in vitro 'neutralizing' anti-IL-2 mAbs; (ii) S4B6 and JES6-1 occupy different IL-2 sites; (iii) IL-2/S4B6 complexes are CD122 dependent; (iv) IL-2/JES6-1 complexes strictly depend on CD25; (v) S4B6 partly covers the CD25 epitope; (vi) JES6-1 occupies CD122/ $\gamma_{\mathrm{c}}$ binding sites; (vii) S4B6 shows a mild clash with CD122; (viii) JES6-1 occupies CD122/ $\gamma_{c}$-binding sites; (ix) S4B6 induces allosteric changes in the CD122 epitope; and (x) JES6-1 allosterically changes the CD25 epitope of IL-2. In integrating these aspects (Figure 4), it can be proposed that association of IL-2 with S4B6 (or S4B6-like mAbs) sterically hinders the binding of CD25 to IL-2 by covering (part of) the CD25-binding site of IL-2 [thus explaining abovementioned points (i) and (v)], which renders IL-2 'blind' to CD25 but dependent on CD122 [point (iii)]. Of note, maybe partial - rather than full - obstruction of the CD25 epitope by S4B6-like mAbs is important for their mechanism of action? Conversely, binding of IL-2 to JES6-1 (or JES6-1-like mAbs) occludes IL-2 residues interacting with CD122 and/or $\gamma_{c}$ [explaining points (ii) and (vi)]; this renders IL-2 dependent on CD25 [point (iv)] and inaccessible to CD122 and/or $\gamma_{c}$ until the IL-2/ 
JES6-1 complex binds to CD25 thus releasing IL-2 from JES6-1 and making it now amenable to CD122 and $\gamma_{c}$.

The proposed allosteric effects in both complexes and the suggested clash between S4B6 and CD122 [13], however, suggest antagonistic forces at work. Moreover, the apparently discordant effects seen in IL-2/S4B6 complexes with increased CD122-binding affinity but decreased STAT5 phosphorylation and reduced T cell proliferation in vitro would suggest further complexity of the system. These aspects require future studies of IL-2 versus IL-2/mAb complexes focusing on the binding kinetics of IL-2 to its receptor subunits, internalization and degradation versus recycling of the IL-2-IL-2R complex, and qualitative and quantitative (i.e., signal duration) assessment of signaling events. Physiologically, binding of IL-2 to the IL-2R and internalization of this quaternary complex leads to the degradation of IL-2, CD122, and $\gamma_{\mathrm{C}}$, while CD25 is recycled [21]. The question arises of whether, in IL-2/mAb complexes, the mAb detaches from IL-2, whether the mAb is internalized or not, and, if the mAb is internalized, whether it is recycled to the cell surface or degraded intracellularly. The former point on dissociation and internalization of the mAb has also practical consequences on whether IL-2/mAb complexes can be devised as single-molecule fusion proteins. Notably, for clinical application, there is some interest in generating a fusion protein of IL-2 complex by using a flexible linker between IL-2 and the $\mathrm{mAb}$, although others do not see a problem with using $\mathrm{IL}-2 / \mathrm{mAb}$ complexes that can dissociate in vivo (Box 1) [10]. Currently, there is only one report describing a fusion protein of the IL-2/S4B6 complex; however, it remains to be tested to what extent this fusion protein still allows association of mlL-2 with S4B6's antigen-binding groove [81]. Notably, it is worth considering the use of S4B6-like mAbs to modulate endogenous IL-2 for selective immune stimulation, as shown in mice [9]; whether similar effects can be achieved with JES6-1-like mAbs remains to be explored.

Similar questions apply to CD25- versus CD122-biased IL-2 muteins, which do or do not require contact with $\mathrm{CD} 25$ or CD122 $/ \gamma_{\mathrm{c}}$. Significantly, a particular issue relevant to IL-2 muteins might be immunogenicity and the generation of antidrug antibodies (see Box 1 for a detailed discussion).

Box 1. Limitations and Immunogenicity of IL-2 Formulations

Immunotherapy using IL-2 suffers from certain shortcomings [6,69]. First, IL-2 exerts both immunosuppressive and immunostimulatory actions by activating Treg cells and cytotoxic effector lymphocytes, including $\mathrm{CD}^{+} \mathrm{T}$ cells and NK cells. Both IL-2/mAb complexes and IL-2 muteins overcome this issue by interfering with interaction of IL-2R subunits with their respective IL-2 epitopes. Second, high doses of IL-2 can lead to VLS via direct binding of IL-2 to and damage of $\mathrm{CD}_{2} 5^{+}$endothelial cells and indirect damage of endothelial cells by NK cell-derived proinflammatory cytokines and vasoactive mediators. This shortcoming is addressed by the lower doses needed of IL-2/mAb complexes and some IL-2 muteins to achieve the same effects as 'wild-type' IL-2 and, in CD122-biased formulations, by avoiding contact with $\mathrm{CD} 25^{+}$pulmonary endothelial cells. Third, $\mathrm{LL}-2$ is short lived in vivo due to its rapid renal clearance, thus necessitating frequent administration. Hence, formulations with longer $T_{1 / 2}$ should be generated by coupling, or complexing, IL-2 to a $\mathrm{mAb}$ (or mAb fragment) specific for IL-2 or a molecule (such as a tumor antigen) enriched in the target tissue [83].

There also exist limitations that are specific to IL-2/mAb complexes or IL-2 muteins. In IL-2/mAb complexes, the administration of IL-2 plus an anti-IL-2 mAb could be seen as potentially problematic for therapeutic application as the two components, although complexed before injection, might dissociate in the patient. This should consider the following. Even if all IL-2/mAb complexes were to dissociate following injection, the amount of IL-2 would reach only $2-5 \%$ of the IL-2 dose usually administered to patients; moreover, the anti-IL-2 mAb could then bind to endogenous IL-2 and direct it to the targeted immune cells. Potentially, IL-2/mAb complexes could be linked by a flexible linker $[10,81]$. With regard to IL-2 muteins, immunogenicity should be considered. Drug immunogenicity is a well-known problem of biological agents [84], including IL-2. Both subcutaneous and intravenous administration of recombinant hIL-2 has been reported to cause anti-IL-2 antibodies in about $50 \%$ of patients; however, neutralizing anti-IL-2 antibodies were seen typically only in 5-10\% of subjects treated [85-87]. However, the N88R IL-2 mutein BAY 50-4798 had already caused antidrug antibodies in $27 \%$ of treated patients after two cycles of intravenous infusion [76]. Hence, it is worth considering strategies to reduce the immunogenicity of IL-2 muteins; for example, by avoiding the formation of aggregates due to hydrophobicity and lack of glycosylation $[85,87]$. 
When aiming for pure CD25- versus CD122-directed IL-2 formulations, one should consider possible antagonistic interactions such as those suggested by the structural data on IL-2/mAb complexes. Notably, the structural data suggest that S4B6 is probably a suboptimal template for an ideal agonistic IL-2/mAb complex, suggesting that S4B6 partly overlaps with CD25's footprint on IL-2 and interferes mildly with CD122 binding. These features would both be unwanted when considering the design of a CD122-directing, high-affinity mimic of CD25 aimed at exerting maximal stimulation of $\mathrm{CD}^{+} \mathrm{T}$ cells while simultaneously blocking $\mathrm{IL}-2$ interaction with $\mathrm{CD} 25^{+}$cells. There, one of the most important challenges will be the setup of a screening system that allows the selection of appropriate anti-IL-2 mAbs (see Outstanding Questions). Furthermore, future structural studies on IL-2/mAb complexes should ideally use S4B6-like hIL-2/mAb complexes tested on human cells and compared with the quaternary IL-2IL-2R complex of the same species.

In conclusion, the study of selective IL-2 formulations has been instrumental in understanding some of the biology of IL-2 and has allowed us dissect the two apparently antagonistic effects of IL-2. This latter finding is perhaps the most important consequence of this research and has led to a conceptual framework for IL-2-based selective immune modulation.

\section{Acknowledgments}

We thank the members of the Boyman laboratory for helpful discussions. This work was funded by Swiss National Science Foundation grant PPOOP3-150751 and Swiss Cancer League grant KFS-3375-02-2014 (both to O.B.), as well as a Candoc fellowship from the University of Zurich (to J.W.).

\section{References}

1. Morgan, D.A. et al. (1976) Selective in vitro growth of T lymphocytes from normal human bone marrows. Science 193, 10071008

2. Robb, R.J. and Smith, K.A. (1981) Heterogeneity of human T-cell growth factor(s) due to variable glycosylation. Mol. Immunol. 18, 1087-1094

3. Taniguchi, T. et al. (1983) Structure and expression of a cloned cDNA for human interleukin-2. Nature 302, 305-310

4. Smith, K.A. (1988) Interleukin-2: inception, impact, and implications. Science 240, 1169-1176

5. Rosenberg, S.A. (2014) IL-2: the first effective immunotherapy for human cancer. J. Immunol. 192, 5451-5458

6. Boyman, O. and Sprent, J. (2012) The role of interleukin-2 during homeostasis and activation of the immune system. Nat. Rev. Immunol. 12, 180-190

7. Klatzmann, D. and Abbas, A.K. (2015) The promise of low-dose interleukin-2 therapy for autoimmune and inflammatory diseases. Nat. Rev. Immunol. 15, 283-294

8. Wang, X. et al. (2005) Structure of the quaternary complex of interleukin-2 with its $\alpha, \beta$, and $\gamma$ c receptors. Science 310, 11591163

9. Boyman, O. et al. (2006) Selective stimulation of T cell subsets with antibody-cytokine immune complexes. Science 311, 1924-1927

10. Rosalia, R.A. et al. (2014) Use of enhanced interleukin-2 formulations for improved immunotherapy against cancer. Curr. Opin. Chem. Biol. 23, 39-46

11. Saadoun, D. et al. (2011) Regulatory T-cell responses to low-dose interleukin-2 in HCV-induced vasculitis. N. Engl. J. Med. 365 , 2067-2077

12. Koreth, J. et al. (2011) Interleukin-2 and regulatory T cells in graftversus-host disease. N. Engl. J. Med. 365, 2055-2066

13. Spangler, J.B. et al. (2015) Antibodies to interleukin-2 elicit selective $\mathrm{T}$ cell subset potentiation through distinct conformational mechanisms. Immunity 42, 815-825

14. Mitra, S. et al. (2015) Interleukin-2 activity can be fine tuned with engineered receptor signaling clamps. Immunity 42, 826-838

15. Yu, A. et al. (2015) Selective IL-2 responsiveness of regulatory $T$ cells through multiple intrinsic mechanisms supports the use of low-dose IL-2 therapy in type 1 diabetes. Diabetes 64, 2172-2183
16. von Spee-Mayer, C. et al. (2015) Low-dose interleukin-2 selectively corrects regulatory $T$ cell defects in patients with systemic lupus erythematosus. Ann. Rheum. Dis. Published online August 31, 2015. http://dx.doi.org/10.1136/annrheumdis-2015-207776 at the interface between tolerance and immunity. Immunity 33 , 153-165

18. Liao, W. et al. (2013) Interleukin-2 at the crossroads of effecto responses, tolerance, and immunotherapy. Immunity 38, 13-25

19. Taniguchi, T. and Minami, Y. (1993) The IL-2/IL-2 receptor system: a current overview. Cell 73, 5-8

20. Letourneau, S. et al. (2009) IL-2- and CD25-dependent immunoregulatory mechanisms in the homeostasis of T-cell subsets. $J$. Allergy Clin. Immunol. 123, 758-762

21. Hemar, A. et al. (1995) Endocytosis of interleukin 2 receptors in human T lymphocytes: distinct intracellular localization and fate of the receptor $\alpha, \beta$, and $\gamma$ chains. J. Cell Biol. 129, 55-64

22. Yu, A. et al. (2000) Efficient internalization of IL-2 depends on the distal portion of the cytoplasmic tail of the IL-2R common $\gamma$-chain and a lymphoid cell environment. J. Immunol. 165, 2556-2562

23. Zelante, T. et al. (2012) Interleukin-2 production by dendritic cells and its immuno-regulatory functions. Front. Immunol. 3, 161

24. O'Shea, J.J. and Paul, W.E. (2010) Mechanisms underlying lineage commitment and plasticity of helper $\mathrm{CD}^{+} \mathrm{T}$ cells. Science $327,1098-1102$

25. Amado, I.F. et al. (2013) IL-2 coordinates IL-2-producing and regulatory T cell interplay. J. Exp. Med. 210, 2707-2720

26. Lenardo, M.J. (1991) Interleukin-2 programs mouse $\alpha \beta$ T lymphocytes for apoptosis. Nature $353,858-861$

27. Van Pariis, L. and Abbas, A.K. (1998) Homeostasis and selftolerance in the immune system: turning lymphocytes off. Science 280, 243-248

28. Wang, H.M. and Smith, K.A. (1987) The interleukin 2 receptor. Functional consequences of its bimolecular structure. J. Exp. Med. 166, 1055-1069

29. Sakaguchi, S. et al. (1995) Immunologic self-tolerance maintained by activated $T$ cells expressing $I L-2$ receptor $\propto$-chains (CD25). Breakdown of a single mechanism of self-tolerance causes various autoimmune diseases. J. Immunol. 155, 1151-1164
17. Malek, T.R. and Castro, I. (2010) Interleukin-2 receptor signaling:

\section{Outstanding Questions}

The conceptual framework of $\mathrm{IL}-2$ based selective immune modulation relies on biased IL-2 formulations. Do similar concepts exist for other cytokines, particularly $\gamma_{c}$ cytokines?

Can the necessity of in vivo testing for appropriate IL-2 complexes (and IL-2 muteins) be circumvented by devising a rational setup of a screening system that allows the selection of appropriate anti-IL-2 mAbs (and muteins)?

In immunotherapy, do we need to target IL-2 to a certain site and, if so, is it the secondary lymphoid organs, the target tissue, or both?

Is binding of IL-2 to its receptor subunits different in IL-2, IL-2 complexes, and $\mathrm{IL}-2$ muteins?

What are the effects on internalization and degradation versus recycling of the IL-2-IL-2R complex on binding of IL-2, IL-2 complexes, or IL-2 muteins?

Do IL-2, IL-2 complexes, and IL-2 muteins lead to different qualitative and quantitative signaling events?

Can an IL-2 complex still exert its selective effects when it is covalently coupled to a mAb specific for $\mathrm{IL}-2$ or another antigen? Can such a molecule still efficiently accumulate, for example at the tumor site, despite its larger size compared with IL-2? 
30. Roediger, B. et al. (2013) Cutaneous immunosurveillance and regulation of inflammation by group 2 innate lymphoid cells. Nat. Immunol. 14, 564-573

31. Krieg, C. et al. (2010) Improved IL-2 immunotherapy by selective stimulation of IL-2 receptors on lymphocytes and endothelial cells. Proc. Natl. Acad. Sci. U.S.A. 107, 11906-11911

32. Mingari, M.C. et al. (1984) Human interleukin-2 promotes proliferation of activated $B$ cells via surface receptors similar to those of activated T cells. Nature 312, 641-643

33. Zubler, R.H. et al. (1984) Activated B cells express receptors for, and proliferate in response to, pure interleukin 2. J. Exp. Med. 160, 1170-1183

34. Wuest, S.C. et al. (2011) A role for interleukin-2 trans-presentation in dendritic cell-mediated T cell activation in humans, as revealed by daclizumab therapy. Nat. Med. 17, 604-609

35. Yu, A. et al. (2009) A low interleukin-2 receptor signaling threshold supports the development and homeostasis of T regulatory cells. Immunity 30, 204-217

36. Malek, T.R. et al. (2002) CD4 regulatory T cells prevent lethal autoimmunity in IL-2R $\beta$-deficient mice, Implications for the nonredundant function of IL-2. Immunity 17, 167-178

37. Sharma, R. et al. (2007) A regulatory $T$ cell-dependent novel function of CD25 (IL-2R $\propto$ ) controlling memory $\mathrm{CD}^{+} \mathrm{T}$ cell homeostasis. J. Immunol. 178, 1251-1255.

38. Williams, M.A. et al. (2006) Interleukin-2 signals during priming are required for secondary expansion of $\mathrm{CD} 8^{+}$memory T cells. Nature $441,890-893$

39. Boyman, O. et al. (2010) The role of interleukin-2 in memory CD8 cell differentiation. Adv. Exp. Med. Biol. 684, 28-41

40. McKinstry, K.K. et al. (2014) Effector CD4 T-cell transition to memory requires late cognate interactions that induce autocrine L-2. Nat. Commun. 5, 5377

41. Long, S.A. et al. (2012) Rapamycin/lL-2 combination therapy in patients with type 1 diabetes augments Tregs yet transiently impairs beta-cell function. Diabetes 61, 2340-2348

42. Hartemann, A. et al. (2013) Low-dose interleukin 2 in patients with type 1 diabetes: a phase 1/2 randomised, double-blind, placebocontrolled trial. Lancet Diabetes Endocrinol. 1, 295-305

43. Matsuoka, K. et al. (2013) Low-dose interleukin-2 therapy restores regulatory $\mathrm{T}$ cell homeostasis in patients with chronic graft-versushost disease. Sci. Transl. Med. 5, 179ra143

44. Cunningham-Rundles, C. et al. (1992) Restoration of immunoglobulin secretion in vitro in common variable immunodeficiency by in vivo treatment with polyethylene glycol-conjugated human recombinant interleukin-2. Clin. Immunol. Immunopathol. 64, 46-56

45. Kovacs, J.A. et al. (1996) Controlled trial of interleukin-2 infusions in patients infected with the human immunodeficiency virus. $N$. Engl. J. Med. 335, 1350-1356

46. INSIGHT-ESPRIT Study Group (2009) Interleukin-2 therapy in patients with HIV infection. N. Engl. J. Med. 361, 1548-1559

47. Letourneau, S. et al. (2010) IL-2/anti-IL-2 antibody complexes show strong biological activity by avoiding interaction with IL-2 receptor alpha subunit CD25. Proc. Natl. Acad. Sci. U.S.A. 107, $2171-2176$

48. Jin, G.H. et al. (2008) Combination treatment with IL-2 and anti-IL2 mAbs reduces tumor metastasis via NK cell activation. Int Immunol. 20, 783-789

49. Verdeil, G. et al. (2008) Adjuvants targeting innate and adaptive immunity synergize to enhance tumor immunotherapy. Proc. Natl. Acad. Sci. U.S.A. 105, 16683-16688

50. Tomala, J. et al. (2009) In vivo expansion of activated naive CD8 ${ }^{+} T$ cells and NK cells driven by complexes of IL-2 and anti-IL-2 monoclonal antibody as novel approach of cancer immunotherapy. J. Immunol. 183, 4904-4912

51. Levin, A.M. et al. (2012) Exploiting a natural conformational switch to engineer an interleukin-2 'superkine'. Nature 484, 529-533

52. Redmond, W.L. et al. (2012) Dual anti-OX4O/LL-2 therapy augments tumor immunotherapy via IL-2R-mediated regulation of OX40 expression. PLOS ONE 7, e34467

53. Cho, H.I. et al. (2012) A potent vaccination strategy that circumvents lymphodepletion for effective antitumor adoptive T-cell therapy. Cancer Res. 72, 1986-1995
54. Molloy, M.J. et al. (2009) Cutting edge: IL-2 immune complexes as a therapy for persistent virus infection. J. Immunol. 182, 4512-4515

55. Hamilton, S.E. et al. (2010) IL-2 complex treatment can protect naive mice from bacterial and viral infection. J. Immunol. 185 6584-6590

56. Webster, K.E. et al. (2009) In vivo expansion of T reg cells with IL 2-mAb complexes: induction of resistance to EAE and long-term acceptance of islet allografts without immunosuppression. J. Exp. Med. 206, 751-760

57. Van Gool, F. et al. (2014) Interleukin-5-producing group 2 innate lymphoid cells control eosinophilia induced by interleukin-2 therapy. Blood 124, 3572-3576

58. Vokaer, B. et al. (2013) IL-17A and IL-2-expanded regulatory T cells cooperate to inhibit Th1-mediated rejection of MHC II disparate skin grafts. PLOS ONE 8, e76040

59. Tang, Q. et al. (2008) Central role of defective interleukin-2 production in the triggering of islet autoimmune destruction. Immunity 28, 687-697

60. Liu, R. et al. (2010) Expansion of regulatory T cells via IL-2/anti-IL-2 mAb complexes suppresses experimental myasthenia. Eur. J. Immunol. 40, 1577-1589

61. Lee, S.Y. et al. (2012) Interleukin-2/anti-interleukin-2 monoclonal antibody immune complex suppresses collagen-induced arthritis in mice by fortifying interleukin-2/STAT5 signalling pathways. Immunology 137, 305-316

62. Wilson, M.S. et al. (2008) Suppression of murine allergic airway disease by IL-2:anti-IL-2 monoclonal antibody-induced regulatory T cells. J. Immunol. 181, 6942-6954

63. Feuerer, M. et al. (2009) Lean, but not obese, fat is enriched for a unique population of regulatory $\mathrm{T}$ cells that affect metabolic parameters. Nat. Med. 15, 930-939

64. Dinh, T.N. et al. (2012) Cytokine therapy with interleukin-2/antiinterleukin-2 monoclonal antibody complexes expands $\mathrm{CD}_{4}{ }^{+} \mathrm{CD} 25^{+} \mathrm{Foxp}^{+}{ }^{+}$regulatory $\mathrm{T}$ cells and attenuates development and progression of atherosclerosis. Circulation 126, 1256-1266

65. Villalta, S.A. et al. (2014) Regulatory T cells suppress muscle inflammation and injury in muscular dystrophy. Sci. Transl. Med. 6, 258ra142

66. Donohue, J.H. and Rosenberg, S.A. (1983) The fate of interleukin2 after in vivo administration. J. Immunol. 130, 2203-2208

67. Lotze, M.T. et al. (1985) In vivo administration of purified human interleukin 2, II. Half life, immunologic effects, and expansion of peripheral lymphoid cells in vivo with recombinant IL 2. J. Immunol. $135,2865-2875$

68. Konrad, M.W. et al. (1990) Pharmacokinetics of recombinant interleukin 2 in humans. Cancer Res. 50, 2009-2017

69. Boyman, O. et al. (2006) Potential use of IL-2/anti-IL-2 antibody mmune complexes for the treatment of cancer and autoimmune disease. Expert Opin. Biol. Ther. 6, 1323-1331

70. Phelan, J.D. et al. (2008) Cutting edge: mechanism of enhancement of in vivo cytokine effects by anti-cytokine monoclonal antibodies. J. Immunol. 180, 44-48

71. Polhill, T. et al. (2012) IL-2/L-2Ab complexes induce regulatory $T$ cell expansion and protect against proteinuric CKD. J. Am. Soc. Nephrol. 23, 1303-1308

72. Rickert, M. et al. (2005) The structure of interleukin-2 complexed with its alpha receptor. Science $308,1477-1480$

73. Reem, G.H. et al. (1985) Induction and upregulation by interleukin 2 of high-affinity interleukin 2 receptors on thymocytes and T cells. Proc. Natl. Acad. Sci. U.S.A. 82, 8663-8666

74. Shanafelt, A.B. et al. (2000) A T-cell-selective interleukin 2 mutein exhibits potent antitumor activity and is well tolerated in vivo. Nat Biotechnol. 18, 1197-1202

75. Laurent, J. et al. (2013) T-cell activation by treatment of cancer patients with EMD 521873 (Selectikine), an IL-2/anti-DNA fusion protein. J. Transl. Med. 11, 5

76. Margolin, K. et al. (2007) Phase I trial of BAY 50-4798, an interleukin-2-specific agonist in advanced melanoma and renal cancer. Clin. Cancer Res. 13, 3312-3319

77. Hu, P. et al. (2003) Generation of low-toxicity interleukin-2 fusion proteins devoid of vasopermeability activity. Blood 101, 4853-4861 


\section{Trends in Immunology}

78. Carmenate, T. et al. (2013) Human IL-2 mutein with higher antitumor efficacy than wild type IL-2. J. Immunol. 190, 6230-6238

79. Klein, C. et al. (2013) Tumor-targeted, engineered IL-2 variant (IL2 v)-based immunocytokines for the immunotherapy of cancer [abstract]. Proceedings of the 104th Annual Meeting of the American Association for Cancer Research. Cancer Res. 73, 486

80. Ardolino, M. et al. (2014) Cytokine therapy reverses NK cell anergy in MHC-deficient tumors. J. Clin. Invest. 124, 4781-4794

81. Tomala, J. et al. (2013) Chimera of IL-2 linked to light chain of antiIL-2 mAb mimics IL-2/anti-IL-2 mAb complexes both structurally and functionally. ACS Chem. Biol. 8, 871-876

82. Arkin, M.R. et al. (2003) Binding of small molecules to an adaptive protein-protein interface. Proc. Natl. Acad. Sci. U.S.A. 100, 1603-1608
83. Hess, C. et al. (2014) Emerging classes of armed antibody therapeutics against cancer. MedChemComm 5, 408-431

84. Boyman, O. et al. (2014) Adverse reactions to biologic agents and their medical management. Nat. Rev. Rheumatol. 10 612-627

85. Allegretta, M. et al. (1986) The development of anti-interleukin-2 antibodies in patients treated with recombinant human interleukin2 (IL-2). J. Clin. Immunol. 6, 481-490

86. Scharenberg, J.G. et al. (1994) The development of anti-interleukin-2 (IL-2) antibodies in cancer patients treated with recombinant IL-2. Eur. J. Cancer 30A, 1804-1809

87. Prummer, O. (1997) Treatment-induced antibodies to interleukin2. Biotherapy 10, 15-24 